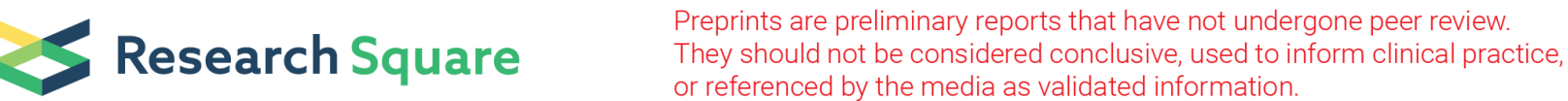

\section{The Mechanism of Ischemic Preconditioning Up- Regulation the Expression of Tissue Kallikrein Protects Neurons from Cerebral Ischemia/Reperfusion Injury}

\section{Lu Su}

Huashan Hospital Fudan University

\section{Dan Liang}

Huashan Hospital Fudan University

\section{Shenyi Kuang}

Huashan Hospital Fudan University

\section{Qiang Dong}

Huashan Hospital Fudan University

Xiang Han ( $\nabla$ hansletter@163.com )

Huashan Hospital Fudan University

Zheng Wang ( $\square$ zheen_wang@163.com )

Huashan Hospital Fudan University

\section{Research}

Keywords: Ischemia/reperfusion, ischemic preconditioning, tissue kallikrein, TK, neuroprotection

Posted Date: May 13th, 2020

DOI: https://doi.org/10.21203/rs.3.rs-27978/v1

License: (c) This work is licensed under a Creative Commons Attribution 4.0 International License.

Read Full License 


\section{Abstract}

Ischemic stroke is an important clinical problem with few effective treatments. Many studies have shown that exogenous tissue kallikrein (TK) can protect neurons against hypoxia/reoxygenation injury. In the present study, we explored the possible molecular mechanisms underlying the regulation and function of endogenous TK. Western blot, chromatin immunoprecipitation (ChIP) and real-time PCR (RT-PCR) revealed that cerebral ischemic preconditioning (IP) upregulated the expression of endogenous TK by regulating the acetylation of histone $\mathrm{H} 3$. Cresyl violet staining was used to assess the neuroprotective role of endogenous TK on rat hippocampal CA1 neurons against cerebral ischemia/reperfusion (I/R) injury. Western blot results showed that IP activated the expression of $\mathrm{p}$-Raf, p-MEK1/2 and p-ERK1/2 by Western blot. Moreover, Western blotfurther determined that endogenous TK upregulated the expression of $\mathrm{p}$-Bad, depressed the release of cytochrome $\mathrm{c}$ and Bax from mitochondria to the cytosol and inhibited caspase-3 activation. In conclusion, endogenous TK can play a neuroprotective role and its upregulation induced by IP treatment can activate the phosphorylation of Raf, MEK1/2 and p-ERK1/2, depress the release of cytochrome $\mathrm{c}$ and Bax from mitochondria to the cytosol and inhibit caspase-3 activation.

\section{Introduction}

Ischemic stroke is a refractory disease with a high incidence, high rate of disability and high mortality [1]. Due to the narrow therapeutic window and insufficiency of neuroprotective treatment for dying neurons $[2,3]$, alternative approaches are urgently needed. Recent years have witnessed great progress in the development of neuroprotective drugs, such as edaravone, a free radical scavenger, argatroban, a direct thrombin inhibitor, butylphthalide, a mitochondrial protective agent and bradykinin receptor agonists of tissue kallikrein (TK).

Among these medicines, TK, a component of the kallikrein-kinin system (KKS), can be self-expressed. In addition, the KKS includes kallikrein, kininogen and bradykinin. Kallikrein includes TK and plasma kallikrein (PK). Bradykinin acts on bradykinin receptor-1 (B1R) and bradykinin receptor-2 (B2R) to regulate mitochondrial energy metabolism and cell cycle progression [4]. Exogenous TK has been shown to achieve a relatively positive clinical effect. If we can reveal the regulatory mechanism of endogenous TK, it may provide a new research direction in relation to protection against ischemic stroke and its treatment.

In 1990, following an investigation in gerbils [5], ischemic tolerance has been regarded as one of the main research directions to discover the mechanism of endogenous neuroprotection. Studies have shown that adenosine [6], the mitochondria [7] and glutamate receptor and its downstream signal transduction pathway are involved in cerebral ischemic tolerance and the associated neuroprotection [8]. Although many neuroscientists have conducted a great deal of research work, the protective mechanism of ischemic tolerance is still unclear. As the KKS is activated after cerebral ischemia/reperfusion (I/R) injury [9], we aimed to investigate the association between the expression of endogenous TK and ischemic preprocessing by establishing an ischemic preconditioning (IP) model. Furthermore, we aimed to detect the function and possible regulatory mechanism of endogenous TK. 
Our previous studies have shown that exogenous TK can inhibit the neurotoxicity induced by glutamate exotoxicity and acidosis after hypoglycaemia and hypoxia through extracellular regulated protein kinase (ERK1/2) in primary cultured neurons [10-12]. Bradykinin receptor-2 belongs to the G-protein coupled receptor family and can activate mitogen-activated protein kinase (MAPK), including ERK1/2, through different mechanisms [13]. There is a serine/threonine domain [14] on the C-terminal of B2R. After phosphorylation, this domain can combine with $\beta$-arrestin-2, forming a stable signal module and promoting the MAPK pathway [15]. It has also been shown that ERK1/2 signalling can affect cell apoptosis and survival through mitochondrial and nuclear pathways. In addition, our previous studies have found that exogenous TK can activate ERK1/2 and its downstream mitochondrial and nuclear pathways through activation of the $\beta$-arrestin-2 assembled B2R-Raf-MEK1/2 signalling module [16]. In this experiment, we further explored the possible signalling pathway of endogenous TK.

\section{Materials And Methods}

\section{Group-division and drug administration of rats}

Male Sprague-Dawley (SD) rats $(250 \pm 20 \mathrm{~g})$ were randomly divided into six groups: sham-operated group, cerebral I/R group, IP-operated group, saline-operated group (saline), antisense oligonucleotides (ODNs) against B2R-operated group (AS-TK) and a selective B2R antagonist HOE 140-operated group (HOE-140). To achieve the I/R models, we induced four-vessel occlusion by occluding the common carotid arteries with aneurysm clips [17]. The rats of the sham-operated group were subjected to the same surgical procedures without inducing I/R. The saline group was injected with normal saline on the basis of the I/R group. The rats of the IP group were given a transient ischemia treatment before cerebral I/R. Moreover, the AS-TK group was injected with antisense ODNs against B2R into the lateral ventricle on the basis of the I/R group and the sequences for B2R AS-ODNs and MS-ODNs used were as follows: AS: 5'CACTGTGGCCGAGATCTACCT-3', 5'-GGCACAACA CCTCTCCAAACA-3'; MS: 5'-AACTGTGGCCGAGAT CTAGGT'-3, 5'-CGCACAACACCTCTCCATTCA-3' [18]. The HOE-140 group was injected with TK receptor B2R inhibitor HOE 140 into the lateral ventricle on the basis of the I/R group, specifically HOE 140 (100 $\mathrm{ug} / \mathrm{kg}$ ) dissolved in $0.9 \% \mathrm{NaCl}$ was injected into the rats i.p. 30 minutes before cerebral ischemia [19]. For each test item, there were six experimental animals.

\section{Animal model of I/R}

Adult male SD rats (250-300 g) obtained from the Shanghai experimental animal centre of the Chinese Academy of Sciences were used throughout testing. The cerebral IP and cerebral I/R models were established by ameliorated four-vessel occlusion, which can effectively decrease the collateral circulation and lead to stable lesions in the hippocampus and cortex.

Anaesthesia was induced with $10 \%$ choral hydrate (350 mg/ $\mathrm{kg}$ i.p.) [20], then the vertebral arteries were electrocoagulated and the common carotid arteries were dissected free. The next day, the bilateral common carotid artery was ligated with aneurysm clips. At this time, the whole brain of the rat was in a state of global cerebral ischemia. The clips were placed for five minutes to induce a short IP. Twenty-four 
hours later, clips were placed for 15 minutes to induce the global ischemic insult. Then the clips were released and the rats were killed at different time points.

The criteria for successful cerebral ischemia included the following: 1) the rats were unconscious and quadriplegic; 2) the righting reflex and corneal reflex had disappeared; and 3) the frequency of the electroencephalogram had slowed. The pre-ischemic model comprised five minutes of global ischemia. Twenty-four hours later, following 15 minutes of global ischemia, the clips were released to restore carotid artery blood flow [21].

Animal care and procedures were performed in accordance with the guidelines of Shanghai Institutes for Biological Sciences of Chinese Academy of Sciences, which was approved by the Department of Laboratory Animal Science, Fudan University (Approval number: 20140143C001).

\section{Immunoblotting}

Immunoblotting analysis was performed according to the method previously described [22] in order to detect the expression of TK protein, the histone H3 acetylation level and the phosphorylation of Raf ( $p$ Raf), MEK1/2 (p-MEK1/2) and ERK1/2 (p-ERK1/2). Proteins were separated using 10\% sodium dodecyl sulphate-polyacrylamide gel electrophoresis (SDS-PAGE) and transferred to polyvinylidene fluoride (PVDF) membranes. After blocking for 1 hour (h), the membranes were incubated overnight with primary antibody in a primary antibody dilution buffer. The membranes were then washed and incubated with secondary antibodies for $1 \mathrm{~h}$ at room temperature. An electrochemiluminescence (ECL) kit was then used to treat the membranes and develop the signal. After immunoblotting, the density of the bands was scanned using Image $\mathrm{J}$ software. We purchased the following primary antibodies from Santa Cruz Biotechnology (Santa Cruz, CA, USA) for our experiments: rabbit polyclonal IgG anti-B2R antibody, mouse monoclonal IgG anti-p-Raf antibody, rabbit polyclonal IgG anti-Raf antibody, mouse monoclonal IgG antip-MEK1/2 antibody, rabbit polyclonal IgG anti-MEK1/2 anti-body, rabbit polyclonal IgG anti-p-ERK1/2 antibody, mouse monoclonal IgG anti-ERK1/2 antibody, goat polyclonal IgG anti- $\beta$-arrestin-2 antibody, mouse monoclonal anti- $\beta$-actin antibody and mouse monoclonal IgG anti-p-Bad antibody. From Cell Signaling Technology (Beverly, MA, USA), we purchased rabbit polyclonal anti-Bax antibody, rabbit polyclonal antibodies anti-NF-KB antibody, rabbit polyclonal anti-cytochrome $\mathrm{c}$ antibody and rabbit polyclonal anticaspase 3 antibody. In addition, we purchased the secondary antibody, goat anti-mouse IgG antibody and goat anti-rabbit IgG antibody from Sigma (Saint Louis, MO, USA).

\section{Chromatin immunoprecipitation}

Chromatin immunoprecipitation (ChIP) assays were performed using a ChIP assay kit (Millipore Corporation, Bedford, MA, USA) to study histone $\mathrm{H} 3$ acetylation binding at the TK promoter. Firstly, DNA and its associated proteins of the peri-infarct tissue were chemically cross-linked by the addition of $37 \%$ formaldehyde ( $1 \%$ final concentration) for 15 minutes at $37^{\circ} \mathrm{C}$. The cross-link reaction was then quenched with glycine $(0.125 \mathrm{~mol} / \mathrm{I}$ final concentration) for five minutes. The DNA was sheared to a size between 200 and 1000 base pairs, cell debris was removed by centrifugation and supernatants were diluted with ChIP dilution buffer. An aliquot of chromatin which was not incubated with antibody was 
used as the input control sample. The remainder was subjected to pre-clearing by incubation with Protein A Sepharose beads for 30 minutes at $4{ }^{\circ} \mathrm{C}$, followed by immunoprecipitation overnight using antibody against acetylated histone H3 (Abcam, Cambridge, MA, USA). Immune complexes were collected by incubation with Protein A Sepharose beads for $1 \mathrm{~h}$ at $4{ }^{\circ} \mathrm{C}$. After a series of sequential washes, bound complexes were eluted from the beads by vortexing in an elution buffer containing $1 \%$ SDS and $0.1 \mathrm{~mol} / \mathrm{I}$ $\mathrm{NaHCO} 3$ and crosslinking was reversed by incubation overnight at $65^{\circ} \mathrm{C}$. Finally, purified DNA samples were subjected to quantitative real-time polymerase chain reaction (qRT-PCR) analyses.

\section{Gene expression analysis}

Reverse transcription PCR (RT-PCR) was performed to evaluate the relative gene expression levels of TK using SYBR Green-based reagents.

The RNA samples were extracted using a MiniBEST Universal RNA Extraction Kit (TaKaRa, Dalian, China) and purified after eliminating genomic DNA with gDNA Eraser (TaKaRa, Dalian, China). The samples were then reverse transcribed into cDNA using the PrimeScriptRT reagent Kit and qRT-PCR was performed using a ViiA ${ }^{\mathrm{TM}} 7$ system. All assays were performed in triplicate with three independent biological RNA preparations. The cycle threshold $(\mathrm{Ct})$ method for the relative quantification of gene expression was used (ddCt) and the normalized $\mathrm{Ct}$ values were calibrated against the control (the sham group) in each experiment.

\section{Histology analysis}

The SD rats were perfusion-fixed with $4 \%$ paraformaldehyde after five days of I/R. After fixation in $4 \%$ paraformaldehyde overnight, the brain tissue was stored in $15 \%$ sucrose for $24 \mathrm{~h}$ and in $30 \%$ sucrose for another $36 \mathrm{~h}$. The brain tissue was dipped into paraffin for $4 \mathrm{~h}$ at $62^{\circ} \mathrm{C}$, before being sectioned into $15 \mu \mathrm{m}$ thick sections. Cryostat sections were prepared and stained with cresyl violet before examination with a light microscope. Moreover, the neuronal density of the hippocampal CA1 pyramidal cells was represented by the number of CA1 pyramidal cells counted under a light microscope (X400).

\section{Statistical analysis}

Statistical analyses were performed with SPSS 17.0 statistical software (SPSS Chicago, IL, USA). Group differences were analysed using a one-way analysis of variance followed by the Duncan (Duncan's new multiple range) post hoc test. $P$ values less than 0.05 were considered significant.

\section{Results}

\section{Cerebral IP upregulates expression of endogenous TK}

To investigate the effect of IP on the regulation of endogenous TK expression, the rats were killed at $3 \mathrm{~h}$, $6 \mathrm{~h}, 12 \mathrm{~h}, 24 \mathrm{~h}, 36 \mathrm{~h}, 48 \mathrm{~h}$ and $72 \mathrm{~h}$ after ischemia. We examined the mRNA level of TK in the hippocampal CA1 area and found that the expression of endogenous TK mRNA increased over time in the IP group, reached its peak at $12 \mathrm{~h}$ and then gradually decreased (Fig. 1A). Furthermore, the immunoblotting results 
showed that the expression of TK protein was significantly increased at $6 \mathrm{~h}, 12 \mathrm{~h}$ and $24 \mathrm{~h}$ in the IP group (Fig. 1B, C).

\section{The upregulation of endogenous TK plays a neuroprotective role in cerebral I/R injury}

We randomly divided the SD rats into six groups: a sham-operated group, cerebral I/R group, IP-operated group, saline-operated group (saline), antisense ODNs against the B2R-operated group (AS-TK) and a selective B2R antagonist HOE-140-operated group (HOE-140). Cresyl violet staining was used to detect the surviving CA1 pyramidal neurons. Normal cells showed round nuclei with pale staining, while the shrunken cells with pyknotic nuclei after reperfusion were counted as dead cells. Cresol violet results showed that IP (Fig. 2A, g, h, had a significant protective effect on the neuronal damage induced by ischemia reperfusion in the hippocampal CA1 region (Fig. 2A, c, d), while antisense ODNs against B2R (Fig. 2A, i, j) or TK receptor inhibitor HOE 140 (Fig. 2A, k, I) were found to significantly antagonize the protective effect. Neuronal densities of the sham-operated group, cerebral I/R group, saline-operated group (saline), IP-operated group, antisense oligonucleotides (ODNs) against B2R-operated group (AS-TK) and a selective B2R antagonist HOE 140-operated group (HOE-140) were $227 \pm 35.21,57.10 \pm 8.12,61.79$ $\pm 9.11,113.11 \pm 16.17,76.24 \pm 9.19,71.98 \pm 10.17$, respectively (Fig. 2B).

\section{Cerebral IP upregulates histone $\mathrm{H} 3$ acetylation}

To detect the effect of ischemia on the modification of histone binding by acetylation, the rats were divided into a sham-operated group, cerebral I/R group, saline-operated group (saline) and IP-operated group. The rats were then killed at different time points after ischemia. Western blot results showed that the expression of acetylated histone $\mathrm{H} 3$ in nuclei increased significantly at $6 \mathrm{~h}, 12 \mathrm{~h}$ and $24 \mathrm{~h}$ after ischemia (Fig. 3A, B). In addition, the chromatin immunoprecipitation results showed that, in the IP group, $\mathrm{H} 3$-acetylated ChIP exhibited obvious enrichment for the TK promoter region at 12 and $24 \mathrm{~h}$ after ischemia (Fig. $3 \mathrm{C}$ ), suggesting that cerebral IP could upregulate the acetylation of histone H3 in the TK promoter region and affect the expression of TK.

\section{Ischemic preconditioning activates the phosphorylation of Raf, MEK1/2 and ERK1/2}

To investigate the downstream signalling pathway of endogenous TK, the rats were divided into a sham group, cerebral I/R group, saline-operated group (saline) and IP-operated group, and then killed at different times following ischemia. Changes in $\mathrm{p}$-Raf, $\mathrm{p}$-MEK1/2 and $\mathrm{p}$-ERK $1 / 2$ were explored by immunoblotting. As shown in Fig. $4 A$ and $B$, the phosphorylation level of Raf, MEK1/2 and ERK1/2 was significantly upregulated after $12 \mathrm{~h}$ in the IP group compared with the I/R group. 


\section{Ischemic preconditioning upregulates p-Bad expression, decreases caspase- 3 activation and inhibits release of cytochrome $\mathrm{c}$ and Bax}

We detected how IP regulated the p-Bad, cytochrome $\mathrm{c}$ and Bax levels in the cytosolic and mitochondrial fraction. As shown in Fig. 5A, the level of p-Bad in the IP group significantly increased compared with the saline-operated and I/R groups. As shown in Fig. 5B and C, IP obviously depressed the release of cytochrome $\mathrm{c}$ and Bax from the mitochondria induced by cerebral I/R in comparison to the saline and cerebral I/R groups. Furthermore, we explored the changes of $\mathrm{p}$-Bad and caspase- 3 in different groups. As shown in Fig. 5A, IP obviously increased the expression of p-Bad and depressed the activation of caspase-3 in comparison to the saline-operated group and the cerebral I/R group. These results indicate that endogenous TK significantly upregulated the expression of $p$-Bad, inhibited the release of cytochrome $\mathrm{c}$ and Bax from mitochondria to the cytosol and depressed the activation of caspase-3.

\section{Discussion}

Stroke is a global health problem. The limitation of the thrombolytic time window makes the development of new neuroprotective agents against the neuronal cell death and neurological deficits caused by ischemic stroke necessary. In the first experiment, we established a IP model to further investigate the regulation mechanism of endogenous TK and found that IP can significantly upregulate the expression of endogenous TK and confer neuroprotection. We then studied the functional mechanism of endogenous TK in the next experiment.

Hypoxia and hypoxia-ischemia have extensive and far-reaching effects on gene expression and protein synthesis. Epigenetic alternation plays an important role in the changes of gene expression induced by ischemia and hypoxia [23]. At present, the research on epigenetic regulation mainly focuses on DNA CG island methylation modification, histone methylation and acetylation modification. Studies have reported that the methylation of DNA CG islands and the histone H3K9 locus can modify the expression of silent genes, while the acetylation of histone and the methylation of histone H3K4 sites activate gene expression [24-26]. Our previous studies have also shown that hypoxia can regulate gene expression by regulating histone methylation and acetylation binding to the neprilysin (NEP) promoter region $[27,28]$. In this experiment, we discovered that IP can upregulate the expression of endogenous TK by affecting histone $\mathrm{H} 3$ acetylation modification. In addition, we found the first association between histone acetylation modification and the expression of endogenous TK. In recent years, many studies have found that histone deacetylase inhibitors injected at different time points can play a neuroprotective role against cerebral I/R injury in rats $[29,30]$. Combined with our research, it is possible that there are similarities between IP and histone deacetylase inhibitors to some extent.

The neuroprotective effect of IP is not a new proposition. Many studies have attempted to achieve a neuroprotective effect by inducing transient ischemia. In addition to a cerebral ischemia tolerance model, 
remote ischemic preconditioning (RIPC) can also provide neuroprotection. On the one hand, it can reduce brain oedema and the volume of cerebral infarction; on the other hand, it can inhibit neuronal apoptosis in the ischemic penumbra. Recently, our study [31] demonstrated that remote ischemic post-conditioning (RIPostC) can promote motor function recovery and reduce brain injury in rats with acute cerebral ischemia by upregulating endogenous TK in a cerebral I/R model. Combined with the results of this experiment, maybe it is possible to find that remote ischemic preconditioning can upregulate the expression of TK, thus protecting the brain against injury in rats. In the future, it may also be possible to conduct a series of clinical trials of ischemic preconditioning in a non-invasive way in a high-risk population of stroke patients, so as to explore whether ischemic preconditioning can reduce the incidence or show beneficial effects in patients with stroke. However, research in this area is highly difficult. Because animal models often cannot represent the real clinical situation after stroke due to a lack of comorbidities, the study design is highly important.

Currently, exogenous TK in the acute phase administered by intravenous injection has achieved more positive clinical outcomes. Recent studies have shown that exogenous TK could prevent I/R-induced neuronal injury via the B2R-ERK1/2 pathway $[10,32]$. Our latest research results also show that exogenous TK can activate ERK1/2 and its downstream mitochondrial pathway through activation of the $\beta$-arrestin-2 assembled B2R-Raf-MEK1/2 signalling module [16]. To explore the signalling pathway of endogenous TK, we investigated the related activation of signal factors after IP. We found that the phosphorylation of Raf, MEK1/2 and ERK1/2 was upregulated after IP and that IP further upregulated the expression of $\mathrm{p}$-Bad, depressed the release of cytochrome $\mathrm{c}$ and Bax from mitochondria to the cytosol and inhibited caspase-3 activation. Combined with the increase of endogenous TK after IP, we have reason to believe that endogenous TK can participate in the activation of Raf, MEK1/2, ERK1/2, affect the mitochondrial pathway, inhibit apoptosis and play a neuroprotective role.

It is a pity that our research on the origin of endogenous TK is not sufficiently thorough. As we can see, cresol violet staining cannot stain neurons exclusively, but also stains glial cells. The acetylation of histone $\mathrm{H} 3$ is also closely related to oligodendrocytes. Accordingly, in our future work we will further explore the source of endogenous TK from the cellular level.

\section{Conclusion}

Ischemic preconditioning can play a neuroprotective role through the upregulation of the expression of endogenous TK by enhancing the acetylation modification of histone H3. This effect is connected to the upregulation of p-Raf, p-MEK1/2 and p-ERK1/2, depressing the release of cytochrome $c$ and Bax from mitochondria to the cytosol and inhibiting caspase-3 activation.

\section{Declarations}

\section{Authors' contributions}


LS, DL,XH and ZW designed the research, performed the analyzed the data, and wrote the paper. LS, DL, SYK and QD performed experiments. All authors reviewed the results and approved the final version to be submitted.

\section{Funding}

This work was supported by the National Natural Science foundation of China (No. 81501134).

\section{Availability of data and materials}

The datasets analyzed during the current study available from the corresponding author upon request.

\section{Ethics approval and consent to participate}

All animal use and experimental protocols were approved by the Department of Laboratory Animal Science, Fudan University (Approval number: 20140143C001).

\section{Consent for publication}

Not applicable.

\section{Competing interests}

The authors declare no competing interests.

\section{References}

1. Feigin VL, Norrving B, Mensah GA. Global burden of stroke. Circulation research. 2017;120(3):43948.

2. Fann DY-W, Lee S-Y, Manzanero S, Chunduri P, Sobey CG, Arumugam TV. Pathogenesis of acute stroke and the role of inflammasomes. Ageing Res Rev. 2013;12(4):941-66.

3. Kaur H, Sarmah D, Saraf J, Vats K, Kalia K, Borah A, et al. Noncoding RNAs in ischemic stroke: time to translate. Ann N Y Acad Sci. 2018;1421(1):19-36.

4. Emanueli C, Madeddu P. Human tissue kallikrein: a new bullet for the treatment of ischemia. Curr Pharm Design. 2003;9(7):589-97.

5. Durukan A, Tatlisumak T. Preconditioning-induced ischemic tolerance: a window into endogenous gearing for cerebroprotection. Experimental translational stroke medicine. 2010;2(1):2. 
6. Pateliya BB, Singh N, Jaggi AS. Possible role of opioids and KATP channels in neuroprotective effect of postconditioning in mice. Biological Pharmaceutical Bulletin. 2008;31(9):1755-60.

7. Abas F, Alkan T, Goren B, Taskapilioglu O, Sarandol E, Tolunay S. Neuroprotective effects of postconditioning on lipid peroxidation and apoptosis after focal cerebral ischemia/reperfusion injury in rats. Turkish Neurosurg. 2010;20(1):1-8.

8. Miao B, Yin X, Pei D, Zhang Q, Zhang G-Y. Neuroprotective effects of preconditioning ischemia on ischemic brain injury through down-regulating activation of JNK1/2 via N-methyl-D-aspartate (NMDA) receptor mediated Akt1 activation. Journal of Biological Chemistry. 2005.

9. Chao J, Chao L. Kallikrein-kinin in stroke, cardiovascular and renal disease. Exp Physiol. 2005;90(3):291-98.

10. Liu L, Zhang R, Liu K, Zhou H, Tang Y, Su J, et al. Tissue kallikrein alleviates glutamate-induced neurotoxicity by activating ERK1. J Neurosci Res. 2009;87(16):3576-90.

11. Liu L, Liu H, Yang F, Chen G, Zhou H, Tang M, et al. Tissue kallikrein protects cortical neurons against hypoxia/reoxygenation injury via the ERK1/2 pathway. Biochem Biophys Res Commun. 2011;407(2):283-87.

12. Su J, Tang Y, Liu L, Zhou H, Dong Q. Regulation of acid-sensing ion channel 1a function by tissue kallikrein may be through channel cleavage. Neurosci Lett. 2011;490(1):46-51.

13. Rozengurt E. Mitogenic signaling pathways induced by $G$ protein-coupled receptors. Journal of cellular physiology. 2007;213(3):589-602.

14. Blaukat A, Pizard A, Breit A, Wernstedt C, Alhenc-Gelas F, Müller-Esterl W, et al. Determination of bradykinin $\mathrm{B} 2$ receptor in vivo phosphorylation sites and their role in receptor function. $\mathrm{J}$ Biol Chem. 2001;276(44):40431-40.

15. Oakley RH, Laporte SA, Holt JA, Caron MG, Barak LS. Differential affinities of visual arrestin, ßarrestin1, and $\beta$ arrestin2 for $\mathrm{G}$ protein-coupled receptors delineate two major classes of receptors. $J$ Biol Chem. 2000;275(22):17201-10.

16. Wang Z, Han X, Cui M, Fang K, Lu Z, Dong Q. Tissue kallikrein protects rat hippocampal CA1 neurons against cerebral ischemia/reperfusion-induced injury through the B2R-Raf-MEK1/2-ERK1/2 pathway. J Neurosci Res. 2014;92(5):651-57.

17. Pulsinelli WA, Brierley JB. A new model of bilateral hemispheric ischemia in the unanesthetized rat. Stroke. 1979;10(3):267-72.

18. Gröger M, Lebesgue D, Pruneau D, Relton J, Kim S-W, Nussberger J, et al. Release of bradykinin and expression of kinin B2 receptors in the brain: role for cell death and brain edema formation after focal cerebral ischemia in mice. Journal of Cerebral Blood Flow Metabolism. 2005;25(8):978-89.

19. Bagaté K, Grima M, De Jong W, Imbs J-L, Barthelmebs M. Effects of icatibant on the ramipril-induced decrease in renal lithium clearance in the rat. Naunyn Schmiedebergs Arch Pharmacol. 2001;363(3):281-87.

20. Su L, Liang D, Kuang S-Y, Dong Q, Han X, Wang Z. Neuroprotective mechanism of TMP269, a selective class IIA histone deacetylase inhibitor, after cerebral ischemia/reperfusion injury. Neural 
regeneration research. 2020;15(2):277.

21. Cheng O, Ostrowski RP, Wu B, Liu W, Chen C, Zhang JH. Cyclooxygenase-2 mediates hyperbaric oxygen preconditioning in the rat model of transient global cerebral ischemia. Stroke. 2011;42(2):484-90.

22. Yi F, Zhang AY, Janscha JL, Li P-L, Zou A-P. Homocysteine activates NADH/NADPH oxidase through ceramide-stimulated Rac GTPase activity in rat mesangial cells. Kidney international. 2004;66(5):1977-87.

23. Yoder JA, Soman NS, Verdine GL, Bestor TH. DNA (cytosine-5)-methyltransferases in mouse cells and tissues. studies with a mechanism-based probe1. Journal of molecular biology. 1997;270(3):38595.

24. Nan X, Ng H-H, Johnson CA, Laherty CD, Turner BM, Eisenman RN, et al. Transcriptional repression by the methyl-CpG-binding protein MeCP2 involves a histone deacetylase complex. Nature. 1998;393(6683):386.

25. Okano M, Bell DW, Haber DA, Li E. DNA methyltransferases Dnmt3a and Dnmt3b are essential for de novo methylation and mammalian development. Cell. 1999;99(3):247-57.

26. Campos AC, Molognoni F, Melo FH, Galdieri LC, Carneiro CR, D'Almeida V, et al. Oxidative stress modulates DNA methylation during melanocyte anchorage blockade associated with malignant transformation. Neoplasia. 2007;9(12):1111-21.

27. Wang Z, Yang D, Zhang X, Li T, Li J, Tang Y, et al. Hypoxia-induced down-regulation of neprilysin by histone modification in mouse primary cortical and hippocampal neurons. PloS one. 2011;6(4):e19229.

28. Wang Z, Zhang XJ, Li T, Li J, Tang Y, Le W. Valproic Acid Reduces Neuritic Plaque Formation and Improves Learning Deficits in APP Swe/PS 1A246E Transgenic Mice via Preventing the Prenatal Hypoxia-Induced Down-Regulation of Neprilysin. CNS Neurosci Ther. 2014;20(3):209-17.

29. Faggi L, Pignataro G, Parrella E, Porrini V, Vinciguerra A, Cepparulo P, et al. Synergistic association of valproate and resveratrol reduces brain injury in ischemic stroke. Int J Mol Sci. 2018;19(1):172.

30. Zhang C, Lu F, Liang J, Zhou Y. Research progress of histone deacetylase 6 inhibitors in the therapy of ischemic stroke. Sheng li xue bao:[Acta physiologica Sinica]. 2018;70(3):301-09.

31. Meng R, Asmaro K, Meng L, Liu Y, Ma C, Xi C, et al. Upper limb ischemic preconditioning prevents recurrent stroke in intracranial arterial stenosis. Neurology. 2012;79(18):1853-61.

32. Liu L, Zhang R, Liu K, Zhou H, Yang X, Liu X, et al. Tissue kallikrein protects cortical neurons against in vitro ischemia-acidosis/reperfusion-induced injury through the ERK1/2 pathway. Exp Neurol. 2009;219(2):453-65.

\section{Figures}




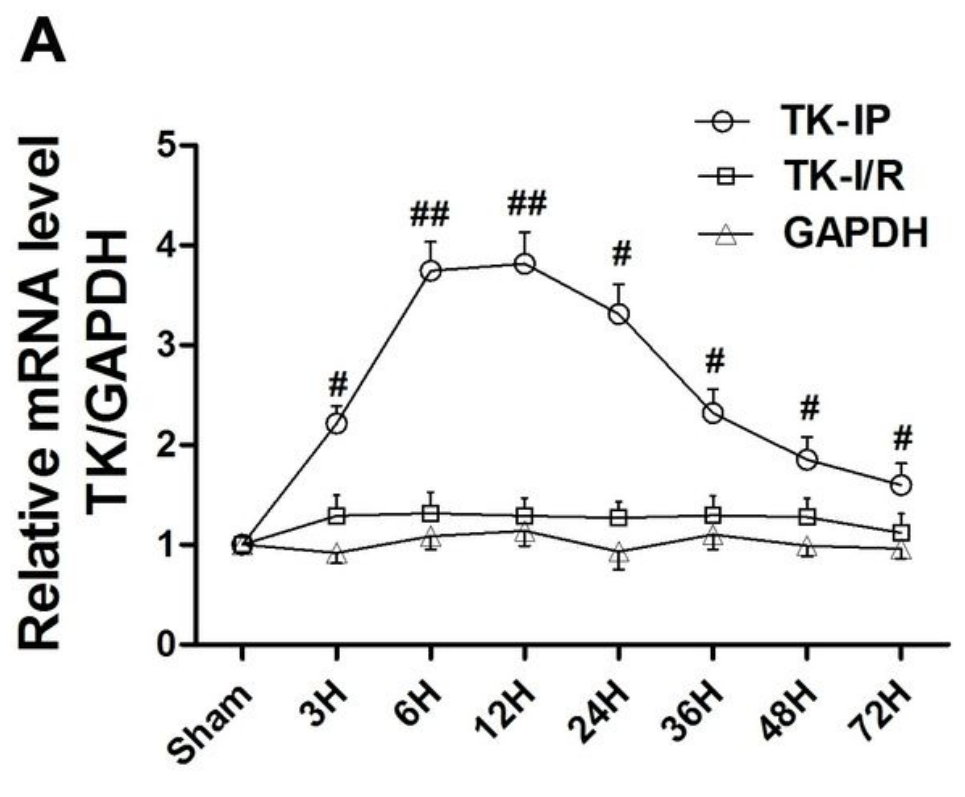

B

Sham $3 \mathrm{H} \quad 6 \mathrm{H} \quad 12 \mathrm{H} \quad 24 \mathrm{H} \quad 36 \mathrm{H} \quad 48 \mathrm{H} \quad 72 \mathrm{H}$

IB:TK
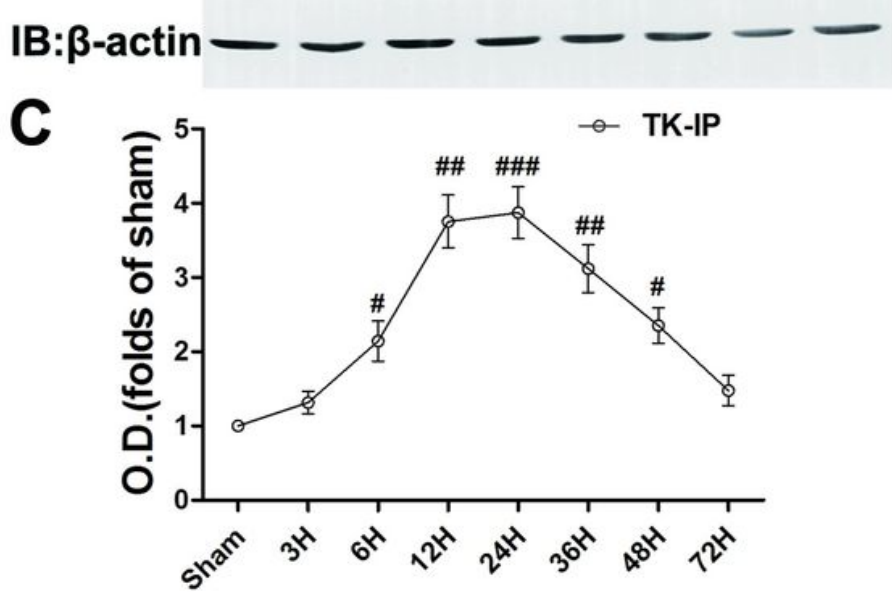

Figure 1

Changes of TK mRNA and TK protein in the hippocampal CA1 region of SD rats after IP. (A): The curve of endogenous TK mRNA over time after IP. $(B, C)$ : The effect of IP on expression TK protein over time. Data are expressed as means \pm SD derived from six independent animals $(n=6)$.
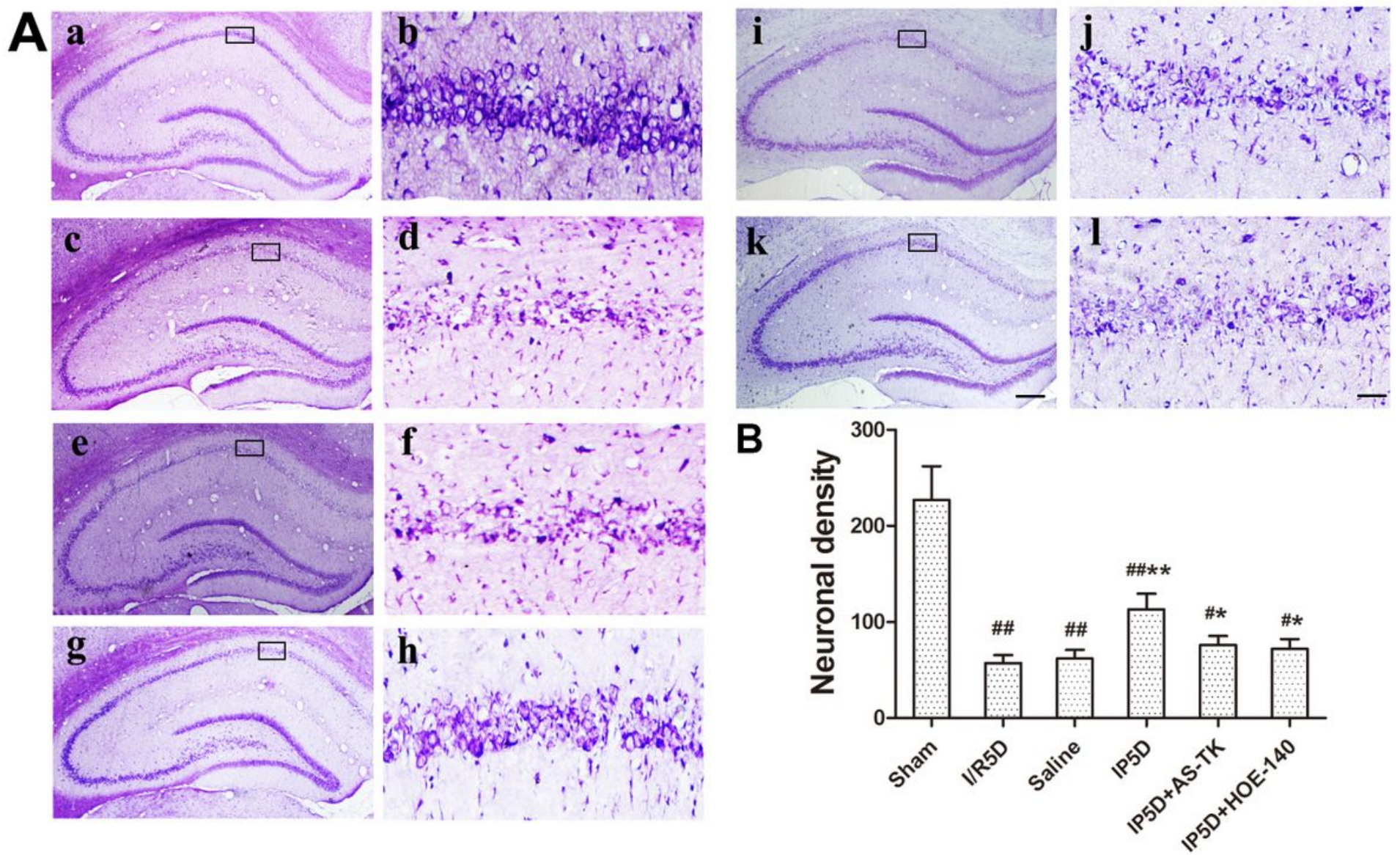

Figure 2 
Effects of IP on neuronal injury by histology analysis. (A, B): Cresyl violet staining was performed on sections from the hippocampus in sham group $(a, b)$, cerebral I/R group $(c, d)$, saline group $(e, f)$, and IP group (g,h), IP combined with antisense ODNs of B2R group (i,j), and IP combined with HOE140 group $(k, l)$. Data are expressed as means $\pm S D$ derived from six independent animals $(n=6)$. \#P $<0.05, \# \# P<$ 0.01 compared with sham group; $* \mathrm{P}<0.05, \star \star \mathrm{P}<0.01$ compared with $\mathrm{I} / \mathrm{R}$ control group. The right columns were magnified versions of the boxes in left columns. a,c,e,g,i,k: x40; b,d,f,h,j,l: $x 400$.

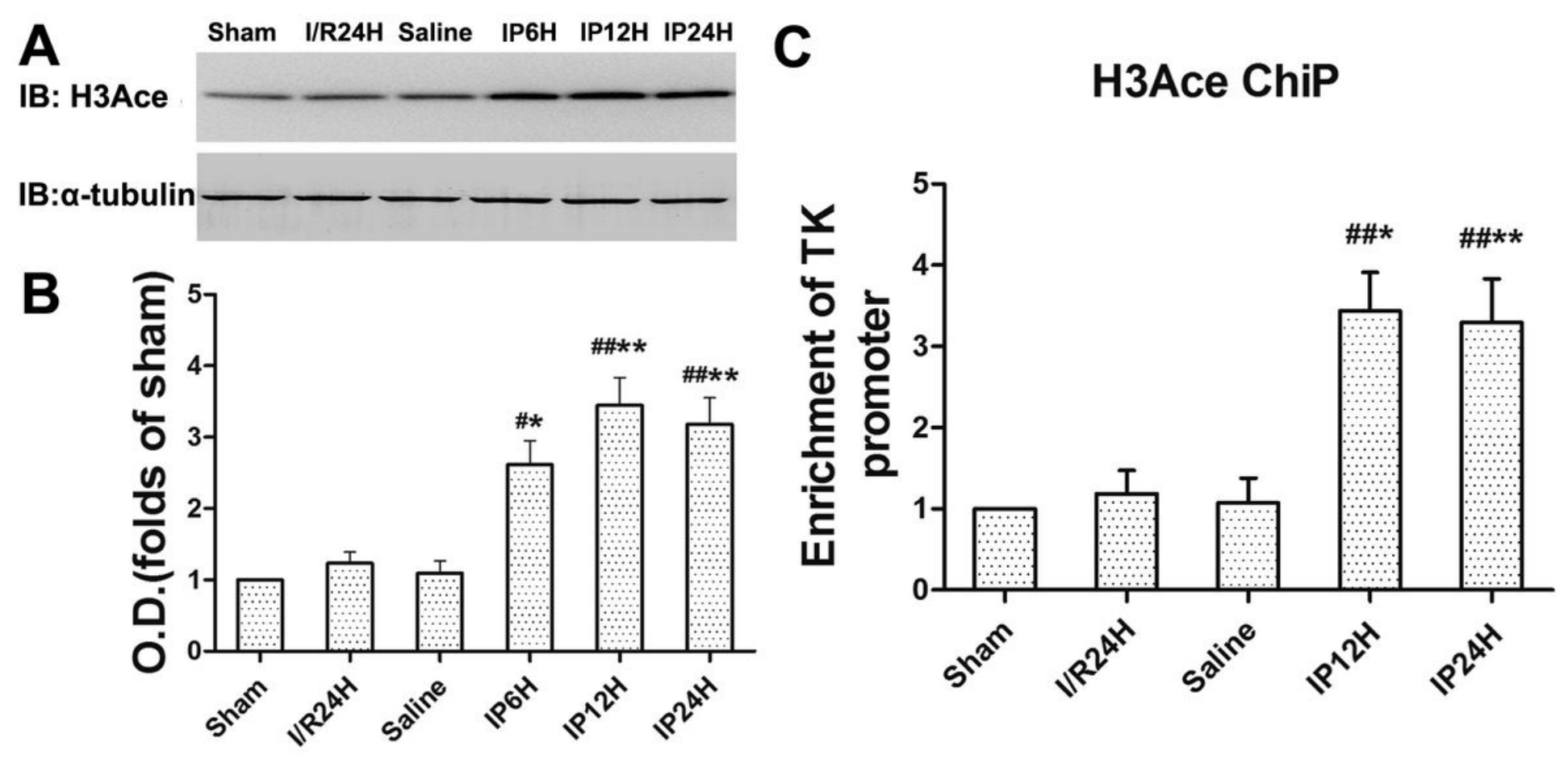

\section{Figure 3}

Cerebral IP upregulates histone H3 acetylation. (A, B): Western Blot analysis showed the upregulation of histone $\mathrm{H} 3$ acetylation after IP compared with I/R group. (C): H3-acetylated ChIP exhibited obvious enrichment for the TK promoter region at 12 and $24 \mathrm{~h}$ after ischaemia. Data are expressed as means \pm SD derived from six independent animals $(n=6)$. \#P< 0.05 , \#\#P<0.01 compared with sham group; $* P<$ 0.05 , ${ }^{*} \mathrm{P}<0.01$ compared with $\mathrm{I} / \mathrm{R}$ control group. 


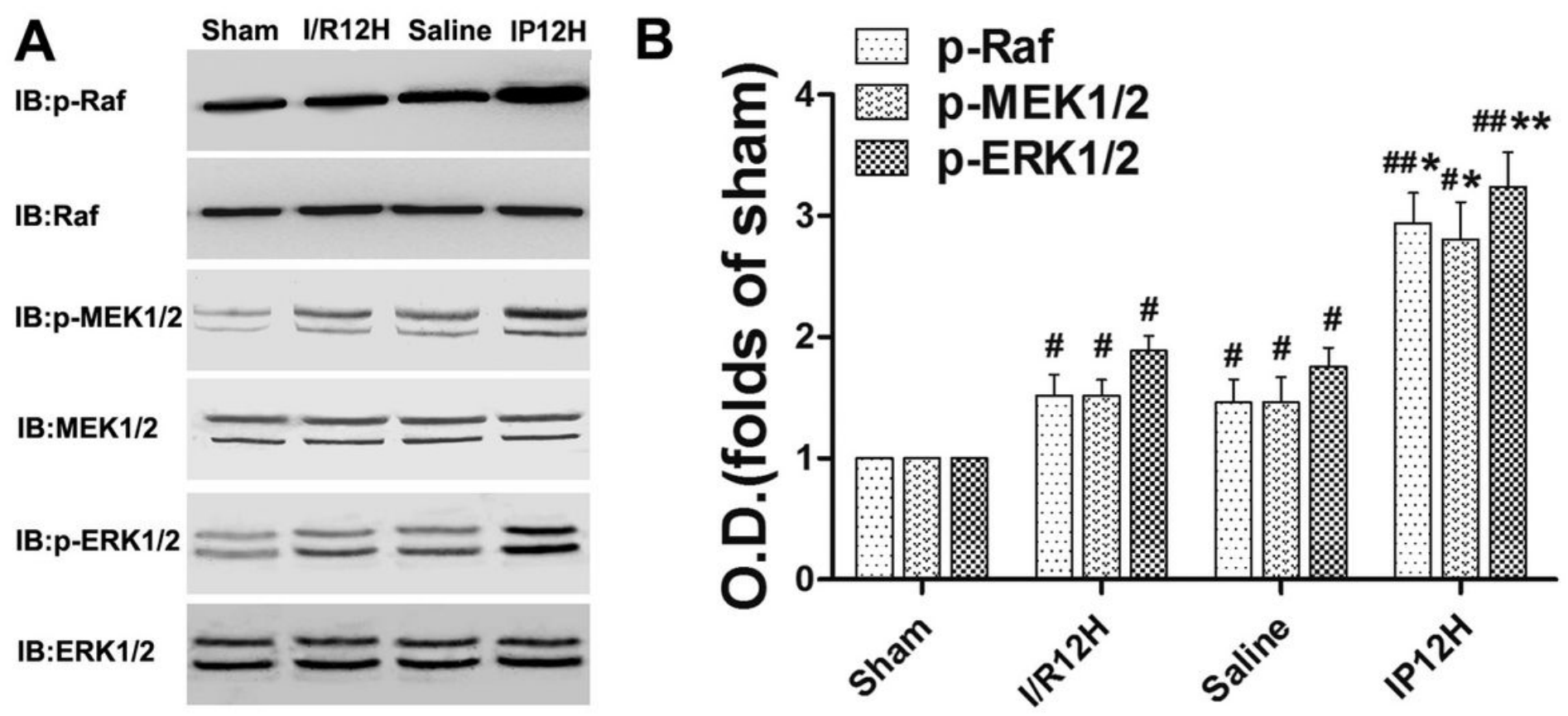

Figure 4

IP activates the phosphorylation of Raf, MEK1/2, and ERK1/2.A,B: IP upregulated the p-Raf, p-MEK1/2 and ERK1/2 level compared with I/ R group. $\beta$-Actin, MEK1/2,ERK1/2 were blotted as controls. Data are expressed as means $\pm S D$ derived from six independent animals $(n=6)$. \#P $<0.05$, \#\#P<0.01 compared with sham group; $* \mathrm{P}<0.05$, $* * P<0.01$ compared with $\mathrm{I} / \mathrm{R}$ control group.

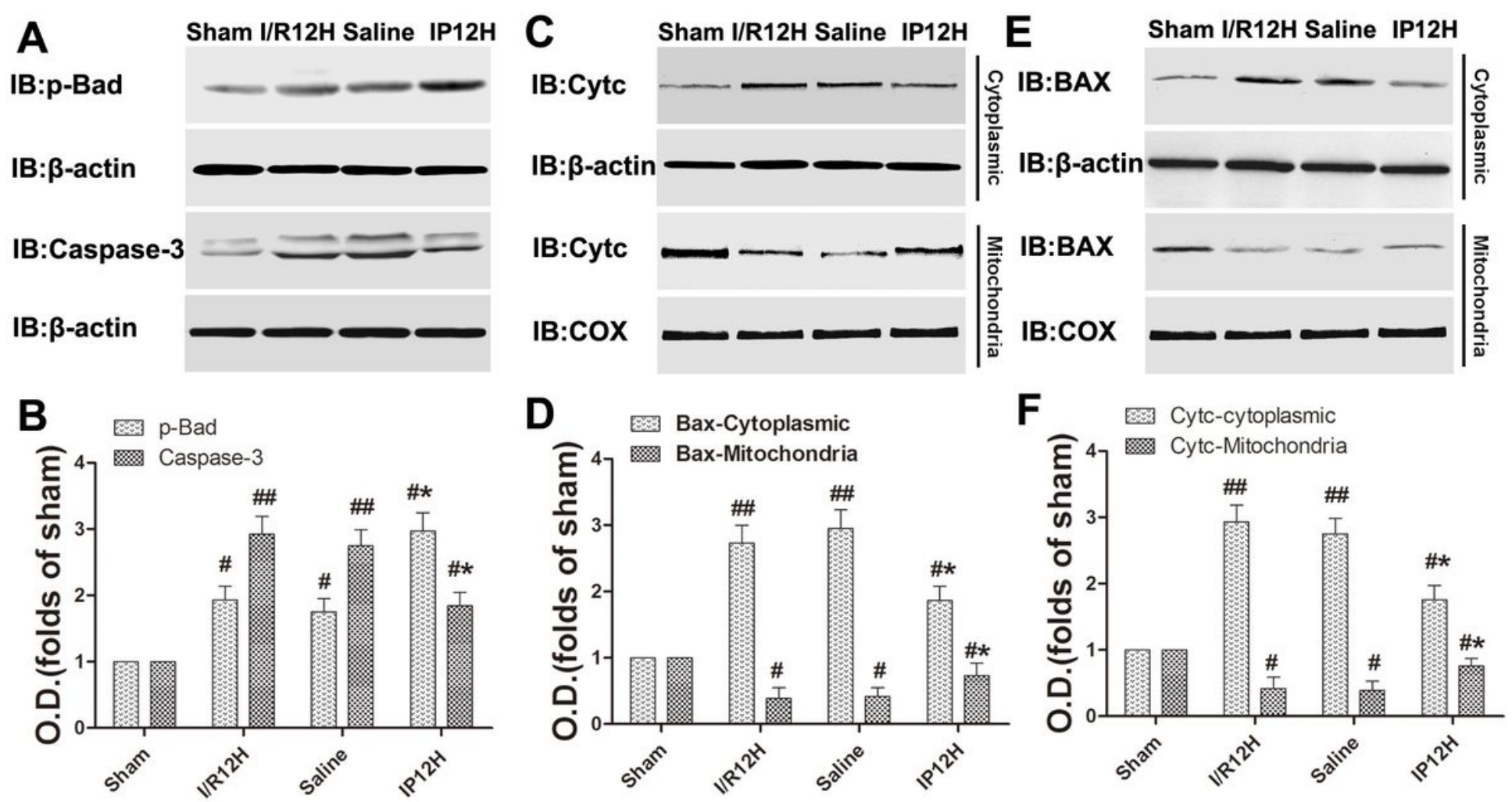

Figure 5 
IP upregulates the expression of P-Bad, depresses the activation Caspase-3, and inhibits the release of cytochrome $c$ and BAX. A, B: IP can increased the expression of $p$-Bad and decreased the activation of Caspase-3 induced by I/R. C, D, E, F: After ischemia-reperfusion, cytc and BAX were transferred from mitochondria to cytoplasm compared with sham group, which could be reduced by ischemic preconditioning. $\beta$-Actin, $C O X$ were blotted as controls. Data are expressed as means $\pm S D$ derived from six independent animals $(n=6)$. \#P $<0.05, \# \# P<0.01$ compared with sham group; ${ }^{*}<0.05, * * P<0.01$ compared with $\mathrm{l} / \mathrm{R}$ control group. 